\title{
Acuidade visual e eletrorretinografia de campo total em pacientes com síndrome de Usher
}

\author{
Visual acuity and full-field electroretinography in patients with Usher's syndrome
}

\author{
Luana Mendieta ${ }^{1}$ \\ Adriana Berezovsky² \\ Solange Rios Salomão ${ }^{3}$ \\ Paula Yuri Sacai ${ }^{4}$ \\ Josenilson Martins Pereira ${ }^{5}$ \\ Sérgio Costa Fantini ${ }^{6}$
}

Trabalho realizado no Laboratório de Eletrofisiologia Visual Clínica do Departamento de Oftalmologia da Universidade Federal de São Paulo - UNIFESP.

${ }^{1}$ Tecnóloga Oftálmica, Pós-Graduanda em Ciências Visuais do Departamento de Oftalmologia da Universidade Federal de São Paulo - UNIFESP. São Paulo (SP).

Professora Adjunta-Doutora do Departamento de Oftalmologia da UNIFESP. São Paulo (SP).

${ }^{3}$ Professora Adjunta-Doutora, Livre Docente, chefe do Laboratório de Eletrofisiologia Visual Clínica do Departamento de Oftalmologia da UNIFESP. São Paulo (SP).

${ }^{4}$ Tecnóloga Oftálmica do Laboratório de Eletrofisiologia Visual Clínica do Departamento de Oftalmologia UNIFESP. São Paulo (SP).

${ }^{5}$ Tecnólogo Oftálmico, Pós-Graduando em Epidemiologia da UNIFESP. São Paulo (SP).

${ }^{6}$ Médico Oftalmologista, Colaborador do Laboratório de Eletrofisiologia Visual Clínica do Departamento de Oftalmologia da UNIFESP. São Paulo (SP).

Apoio: FAPESP no. 97/11493-3 para Solange Rios Salomão.

Endereço para correspondência: Luana Mendieta. R. Botucatu, 822 - São Paulo (SP) CEP 04023-062 E-mail: lmendieta@oftalmo.epm.br

Recebido para publicação em 01.04.2004

Versão revisada recebida em 24.08.2004

Aprovação 03.11.2004

\begin{tabular}{|c|}
\hline RESUMO \\
\hline $\begin{array}{l}\text { A síndrome de Usher (SU) é doença autossômica recessiva caracterizada } \\
\text { por perda auditiva neuro-sensorial acompanhada de retinose pigmentária } \\
\text { (RP). Objetivo: Analisar a eletrorretinografia de campo total (ERG) e a } \\
\text { acuidade visual (AV) em pacientes com síndrome de Usher tipos I e II. } \\
\text { Métodos: Foram estudadas as respostas da eletrorretinografia de campo } \\
\text { total e a acuidade visual de } 22 \text { pacientes (idade média }=26,8 \pm 16,8 \text { anos). } \\
\text { Destes, } 17 \text { tinham síndrome de Usher tipo I e } 5 \text { tinham síndrome de Usher } \\
\text { tipo II. Resultados: A acuidade visual média do grupo síndrome de } \\
\text { Usher I foi de } 0,9 \text { logMAR ( } 20 / 160 \text {, equivalente de Snellen) e do grupo } \\
\text { síndrome de Usher II de } 0,4 \text { logMAR ( } 20 / 50 \text {, equivalente de Snellen). As } \\
\text { respostas dos bastonetes e as máximas respostas mostraram-se não } \\
\text { detectáveis nos dois grupos. A amplitude média dos potenciais } \\
\text { oscilatórios foi de } 14,5 \mu \mathrm{V} \pm 6,1 \text { na síndrome de Usher I e na síndrome de } \\
\text { Usher II de } 12,6 \mu \mathrm{V} \pm 5,2 \text {. As respostas de cones foram não detectáveis em } \\
95 \% \text { dos pacientes com síndrome de Usher Ie em } 100 \% \text { dos pacientes com } \\
\text { síndrome de Usher II. A amplitude média do flicker a } 30 \text { Hz nos pacientes } \\
\text { com síndrome de Usher I foi de } 3,1 \mu \mathrm{V} \pm 4,1 \text { e do tempo de culminação de } \\
34,0 \text { ms } \pm 6,2 ; \text { nos pacientes com síndrome de Usher II a média de amplitude } \\
\text { foi de } 1,0 \text { mV } \pm 0,6 \text { e do tempo de culminação de } 35,8 \text { ms } \pm 3,1 \text { Conclusão: } \\
\text { A acuidade visual mostrou-se relativamente preservada nos dois gru- } \\
\text { pos, porém com melhores resultados no grupo de síndrome de Usher II. } \\
\text { Os achados eletrorretinográficos mostraram-se grandemente reduzidos } \\
\text { em ambos os grupos, com a maioria dos pacientes apresentando respos- } \\
\text { tas não detectáveis de bastonetes e cones. }\end{array}$ \\
\hline
\end{tabular}

Descritores: Retinite pigmentosa; Síndrome de Usher; Eletrorretinografia; Acuidade visual; Eletrofisiologia; Doenças retinianas; Portadores de deficiência visual

\section{INTRODUÇ̃̃̃O}

A síndrome de Usher (SU) foi descrita primeiramente por Von Graefe e, posteriormente, por Liebreich, mas foi Charles Usher, um médico oftalmologista britânico, quem descobriu, em 1914, a hereditariedade da síndrome, que passou a ter seu nome ${ }^{(1)}$. A SU é definida como uma condição autossômica recessiva em que ocorre a associação de deficiências neurosensoriais hereditárias, com perda auditiva, parcial ou total, e diminuição progressiva da visão decorrente da degeneração das células fotorreceptoras da retina, os cones e bastonetes, denominada de retinose pigmentária $(\mathrm{RP})^{(1-2)}$. Nesta doença, os bastonetes são as primeiras células a serem afetadas, levando à cegueira noturna $^{(1-2)}$. A incidência da SU é de 3/100.000 pessoas, enquanto que no grupo de pacientes com deficiência auditiva, a prevalência dessa síndrome é de 3 a $6 \%{ }^{(1-3)}$. Um estudo realizado pelo Grupo Brasil de Apoio ao Surdo-Cego 
e Múltiplo Deficiente Sensorial, recentemente, estimou a existência de 250 pessoas com surdocegueira no país ${ }^{(4)}$.

Atualmente, a classificação mais empregada da síndrome de Usher é a constituída por quatro principais tipos, de acordo com a idade de aparecimento das deficiências e a severidade ${ }^{(1-2)}$. A SU tipo I (SU I) é caracterizada pela perda grave e congênita da audição, ausência da função vestibular e o aparecimento da RP ainda na primeira década de vida; a SU tipo II (SU II) é caracterizada pela perda congênita moderada da audição, função vestibular preservada e início da RP na segunda década de vida; a SU tipo III (SU III) caracteriza-se pela perda auditiva progressiva que tem início tardio, ataxia vestibular, com início da RP na fase da puberdade, podendo apresentar psicose (síndrome de Hallgren); a SU tipo IV (SU IV) é a mais rara, caracterizada pela deficiência auditiva severa, RP e retardo mental ${ }^{(1-2)}$.

O diagnóstico precoce da síndrome de Usher é importante para o planejamento familiar do paciente, bem como re-adaptações apropriadas para o funcionamento do paciente na sociedade $^{(5)}$. Um dos métodos mais usados na rotina clínica oftalmológica para o diagnóstico de RP é a eletrorretinografia de campo total (ERG) ${ }^{(5-7)}$. O ERG é o registro complexo dos potenciais elétricos da retina em resposta à estimulação lumino$\mathrm{sa}^{(5,8)}$. Trata-se de um método que avalia quantitativa e objetivamente a função elétrica das células retinianas e pode preceder alterações fundoscópicas, além de diagnosticar ou excluir patologias retinianas hereditárias ${ }^{(8-9)}$.

Outras funções visuais que têm sido estudadas na SU são a avaliação do campo visual periférico e a medida da acuidade visual. É comum ocorrer constrição do campo visual periférico, devido à perda da função dos bastonetes que estão localizados na periferia da retina, tendo como queixa principal a pobre visão noturna ${ }^{(10-11)}$. Com relação à acuidade visual, estudos mostram acuidade visual de 20/70 ou melhor em aproximadamente $50 \%$ dos casos de $\mathrm{SU}^{(10,12-13)}$.

O objetivo deste estudo é analisar as respostas do ERG de campo total, de acordo com o protocolo padrão recomendado pela International Society for Clinical Electrophysiology of Vision (ISCEV) e os valores da acuidade visual de pacientes com síndrome de Usher dos tipos I e II.

\section{MÉTODOS}

Este estudo foi realizado no Laboratório de Eletrofisiologia Visual Clínica do Departamento de Oftalmologia da Universidade Federal de São Paulo - Escola Paulista de Medicina no período de dezembro de 1998 a abril de 2003, com análise retrospectiva dos prontuários de todos os pacientes com diagnóstico prévio de síndrome de Usher. Este estudo seguiu os princípios da Declaração de Helsinque.

Foram examinados neste período 22 pacientes (12 do sexo feminino e 10 do sexo masculino) com idades variando de 5 a 57 anos (média $=26,8 \pm 16,8$ anos, mediana $=25,0$ ), alocados em dois grupos: SU I - Dezessete pacientes com idades variando entre 5 e 50 anos (média $=23,6 \pm 15,6$ anos, mediana $=21,5$ anos) e SU II - Cinco pacientes com SU do tipo II com idades de 15 a 57 anos (média $=37,0 \pm 18,1$ anos, mediana $=36,0$ anos ).
Grupo síndrome de Usher I: Dezessete pacientes, oito do sexo feminino (idade média $=24,1 \pm 15,0$ anos, mediana $=26,5)$ e dez do sexo masculino (idade média $=22,2 \pm 16,2$ anos, mediana $=18,0)$. A deficiência de visão noturna foi a principal queixa em $16 / 17$ pacientes $(94,1 \%)$ seguida de perda de campo visual periférico em 10/17 casos (58,8\%). A consangüinidade esteve presente em aproximadamente metade dos casos $(9 / 17)$, todos oriundos de pais primos de primeiro grau.

Grupo síndrome de Usher II: Cinco pacientes, quatro do sexo feminino (idade média $=37,3 \pm 20,9$ anos, mediana $=38,5$ ) e um do sexo masculino 36 anos. A deficiência de visão noturna foi a queixa principal em 4/5 pacientes; seguida de perda de campo visual periférico em $2 / 5$ deles. A consangüinidade esteve presente em $2 / 5$, com história de pais primos de primeiro grau.

\section{PROCEDIMENTOS}

A acuidade visual foi medida monocularmente, com a melhor correção óptica, pela tabela ETDRS a 4 metros de distância.

A eletrorretinografia de campo total (ERG) foi realizada após 30 minutos de adaptação ao escuro e dilatação máxima da pupila (diâmetro pupilar mínimo de $6 \mathrm{~mm}$ ). Após esse período, as respostas foram obtidas, utilizando um eletrodo bipolar (GoldLens), em forma de lente de contato, sob anestésico tópico e metilcelulose a $2 \%$ para proteção da córnea. Um eletrodo terra foi colocado no lobo da orelha ipsilateral, preenchido com gel eletrolítico.

O ERG seguiu o protocolo padrão recomendado pela ISCEV, no qual cinco respostas foram registradas após apresentações de estímulos luminosos:

1) Resposta escotópica de bastonetes

2) Resposta escotópica máxima (cones e bastonetes)

3) Resposta escotópica de potenciais oscilatórios

4) Resposta fotópica de cones ao flash único

5) Resposta fotópica de flicker a $30 \mathrm{~Hz}$

Os parâmetros analisados foram as amplitudes das respostas, medidas em microvolts $(\mu \mathrm{V})$ e o tempo de culminação da onda-b, medido em milisegundos (ms). Os valores das amplitudes menores do que $2 \mu \mathrm{V}$ foram considerados não detectáveis (ND). Todos os valores foram comparados com os valores normais, utilizando o mesmo procedimento, padronizados no próprio Laboratório ${ }^{(14)}$.

Os resultados obtidos do ERG, acuidade visual e idade dos dois grupos com síndrome de Usher foram comparados estatisticamente utilizando o teste $\mathrm{t}$ de Student. A acuidade visual desses dois grupos também foi comparada utilizando o teste estatístico Mann-Whitney.

\section{RESULTADOS}

A acuidade visual dos dois grupos foi comparável (Tabela 1). A média dos 15 pacientes que informaram a visão com SU I foi de 0,9 $\log$ MAR (20/160, equivalente de Snellen) e dos 5 
pacientes com SU II de 0,4 logMAR (20/50, equivalente de Snellen), como mostra a tabela 2.

A figura 1 mostra registros do ERG de campo total repre- sentativos de pacientes com síndrome de Usher tipos I e II, respectivamente, juntamente com um exemplo de registro eletrorretinográfico de um adulto jovem normal.

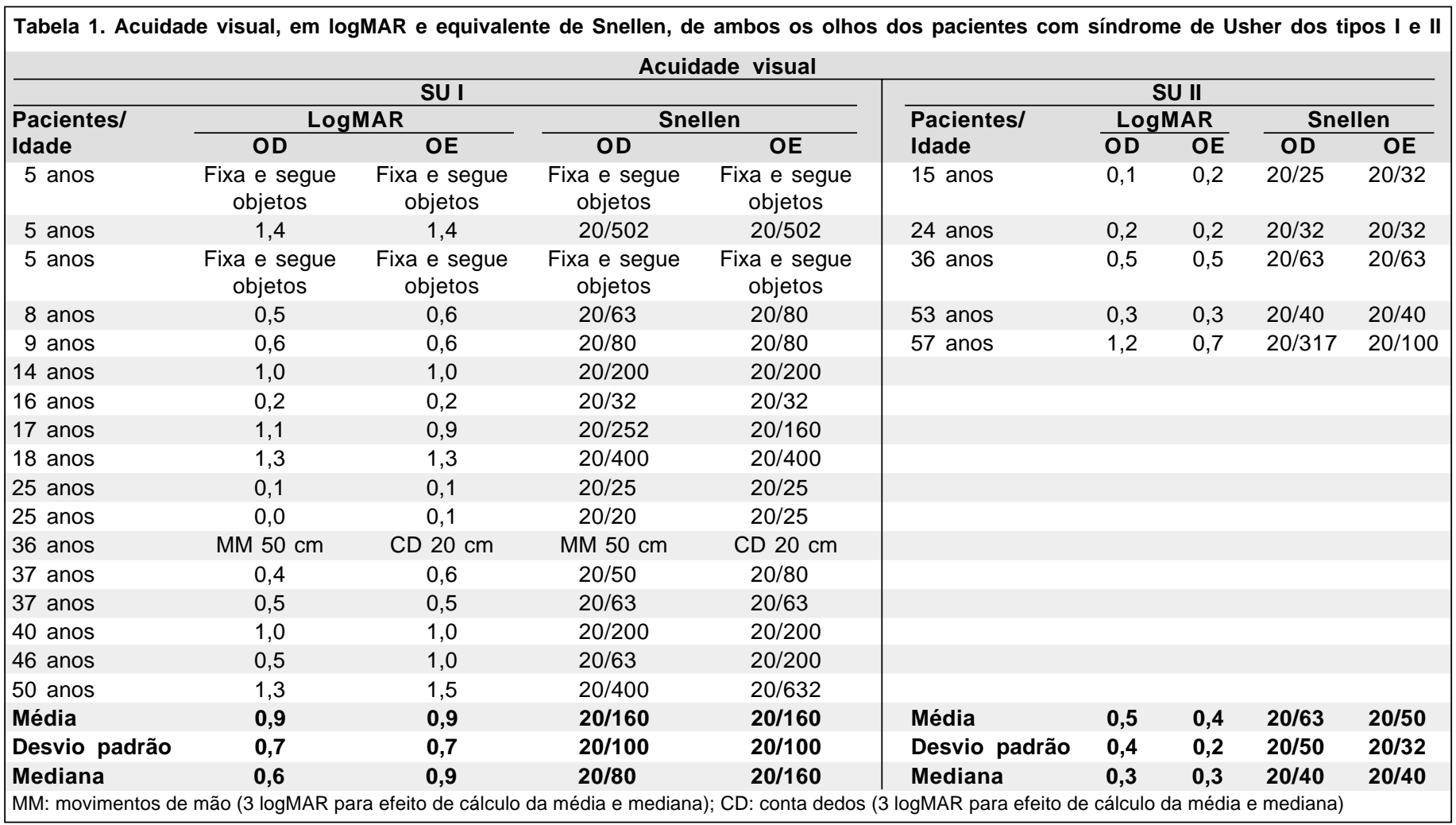

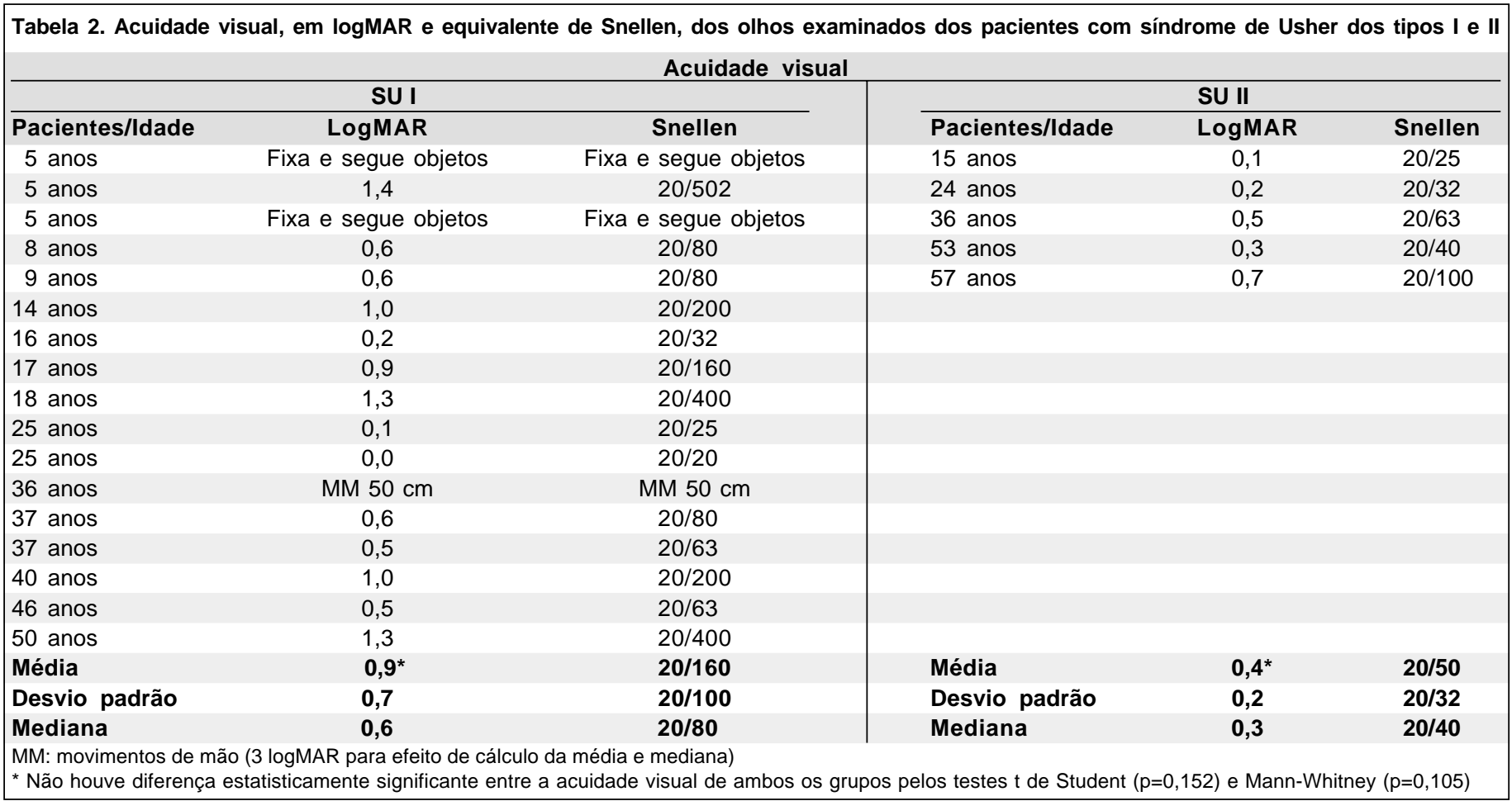




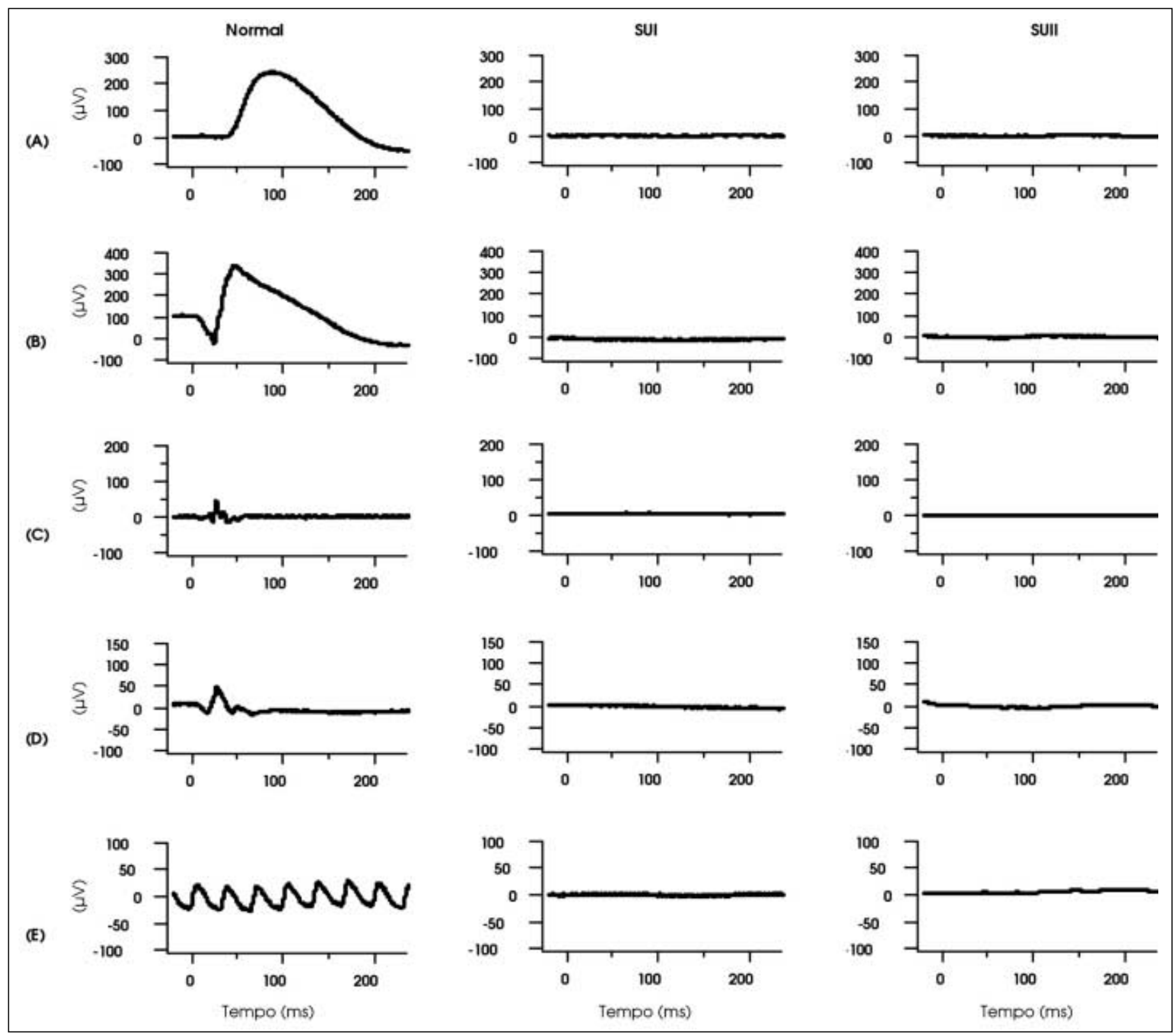

Figura 1 - Respostas eletrorretinográficas de um voluntário adulto jovem normal e de dois pacientes com síndrome de Usher, respectivamente SU I e SU II. As respostas obtidas, segundo o protocolo padrão sugerido pela Sociedade Internacional de Eletrofisiologia Visual Clínica (ISCEV), são: (A) Respostas escotópicas de bastonetes; (B) Respostas escotópicas máxima (cones e bastonetes); (C) Respostas escotópicas dos potenciais oscilatórios; (D) Respostas fotópicas de cones; (E) Respostas fotópicas de flicker $30 \mathrm{~Hz}$. Observa-se respostas não detectáveis do ERG nos pacientes SU I e SU II quando comparadas às respostas do voluntário normal

Os valores de média e desvio padrão para a amplitude $(\mu \mathrm{V}) \mathrm{e}$ o tempo de culminação da onda- $b$ (ms) dos achados eletrorretinográficos estão demonstrados nas tabelas 3 e 4 , respectivamente, comparados com os valores normativos do Laboratório.

As respostas dos bastonetes e as máximas respostas mostraram-se não detectáveis nos dois grupos. A amplitude média dos potenciais oscilatórios foi de $14,5 \mu \mathrm{V} \pm 6,1$ na SU I e na SU II de $12,6 \mu \mathrm{V} \pm 5,2$. As respostas de cones foram não detectáveis em $95 \%$ dos pacientes com SU I e em 100\% dos pacientes com SU II. A amplitude média do flicker a $30 \mathrm{~Hz}$ nos pacientes com SU I foi de $3,1 \mu \mathrm{V} \pm 4,1$ e a média do tempo de culminação foi de
$34,0 \mathrm{~ms} \pm 6,2$; nos pacientes com SU II a média de amplitude foi de $1,0 \mu \mathrm{V} \pm 0,6$ e do tempo de culminação de $35,8 \mathrm{~ms} \pm 3,1$.

A idade dos pacientes do grupo SU II não foi estatisticamente diferente que no grupo SU I.

\section{DISCUSSÃO}

Como esperado, o eletrorretinograma de campo total mostrou ser um exame útil no diagnóstico da síndrome de Usher, mesmo nos pacientes assintomáticos ainda quando jovens ${ }^{(5-6,9,15)}$. 


\begin{tabular}{|c|c|c|c|c|c|c|}
\hline \multicolumn{7}{|c|}{$\begin{array}{l}\text { Tabela 3. Valores das amplitudes }(\mu \mathrm{V}) \mathrm{d} \\
\text { nográficas dos pacientes com síndrom }\end{array}$} \\
\hline \multicolumn{7}{|c|}{ Amplitudes } \\
\hline & Idade & Bastonetes & $\begin{array}{l}\text { Máxima } \\
\text { resposta }\end{array}$ & $\begin{array}{l}\text { Potenciais } \\
\text { oscilatórios }\end{array}$ & Cones & $\begin{array}{l}\text { Flicker } \\
\text { a } 30 \mathrm{~Hz}\end{array}$ \\
\hline \multirow{17}{*}{$\overline{\text { ळ }}$} & 5 a. & ND & ND & 16,3 & ND & 3,7 \\
\hline & 5 a. & ND & ND & 15,4 & 28,5 & 17,9 \\
\hline & 5 a. & ND & ND & 24,0 & ND & 2,1 \\
\hline & 8 a. & ND & ND & 20,1 & ND & ND \\
\hline & 9 a. & ND & ND & 20,8 & ND & 2,5 \\
\hline & $14 \mathrm{a}$. & ND & ND & 7,7 & ND & 6,1 \\
\hline & $16 \mathrm{a}$. & ND & ND & 8,9 & ND & ND \\
\hline & 17 a. & ND & ND & 3,1 & ND & ND \\
\hline & $18 \mathrm{a}$. & ND & ND & 11,7 & ND & 4,9 \\
\hline & 25 a. & ND & ND & 12,1 & ND & ND \\
\hline & $25 \mathrm{a}$. & ND & ND & 8,7 & ND & ND \\
\hline & $36 \mathrm{a}$. & ND & ND & 20,3 & ND & ND \\
\hline & $37 \mathrm{a}$. & ND & ND & 23,1 & ND & 2,4 \\
\hline & $37 \mathrm{a}$. & ND & ND & 7,0 & ND & 2,0 \\
\hline & 40 a. & ND & ND & 17,5 & ND & ND \\
\hline & $46 \mathrm{a}$. & ND & ND & 14,1 & ND & ND \\
\hline & 50 a. & ND & ND & 16,5 & ND & ND \\
\hline \multirow{5}{*}{$=$} & $15 \mathrm{a}$. & ND & ND & 15,9 & ND & ND \\
\hline & $24 \mathrm{a}$. & ND & ND & 18,3 & ND & ND \\
\hline & 36 a. & ND & ND & 14,4 & ND & ND \\
\hline & $53 \mathrm{a}$. & ND & ND & 5,6 & ND & ND \\
\hline & 57 a. & ND & ND & 9,0 & ND & ND \\
\hline
\end{tabular}

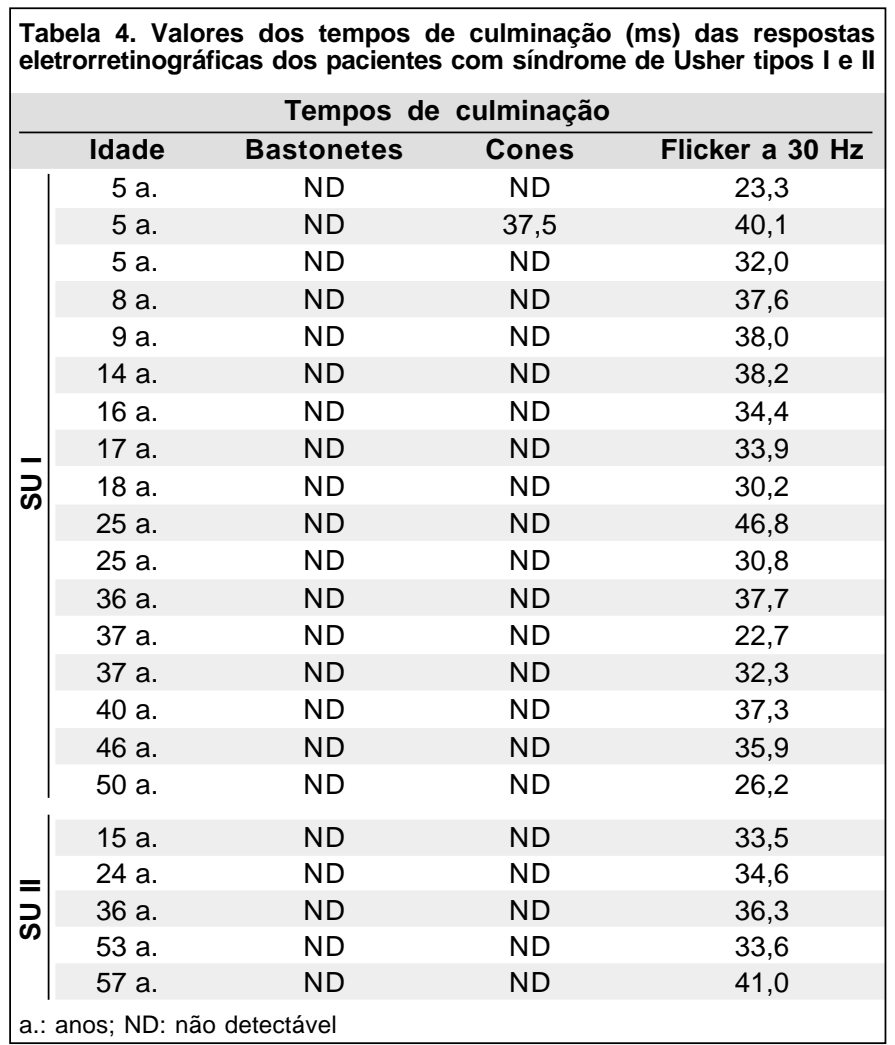

Existe controvérsia na literatura se a severidade dos sintomas oculares e os achados clínicos diferem em relação ao tipo de síndrome de Usher. Alguns autores acreditam que a forma mais severa de RP está relacionada à SU tipo $\mathrm{I}^{(16-17)}$. Mais recentemente, outros estudos relataram que o prognóstico visual não está necessariamente associado ao tipo da $\mathrm{SU}^{(13,18)}$. Em um estudo realizado nos Estados Unidos com 67 pacientes com síndrome de Usher ${ }^{(13)}$ e outro trabalho com 39 pacientes, realizado na Alemanha ${ }^{(18)}$, não encontraram diferença nos dois tipos. Os resultados obtidos no presente estudo confirmaram que a deficiência de visão noturna e o diagnóstico da RP ocorrem em ambas as formas de síndrome de Usher igualmente, não havendo diferença entre os grupos.

As respostas de bastonetes obtidas no ERG de campo total mostraram-se não detectáveis em ambos os grupos. As respostas de cones estavam drasticamente reduzidas tanto para as respostas fotópicas de cones, quanto para a estimulação de flicker a $30 \mathrm{~Hz}$ nos dois grupos.

Alguns autores acreditam que pacientes portadores de RP, associados a síndrome de Usher, são considerados como cegos legais, ou seja, apresentam acuidade visual $\leq 20 / 200$ no melhor olho, com a melhor correção óptica ${ }^{(12,15,19)}$. Entretanto, estudos mais recentes descreveram que, mesmo que a doença esteja avançada, a maioria dos pacientes tem a acuidade visual preservada, isto é, acuidade visual $\geq 20 / 40$ no melhor olho ${ }^{(12-13,18-19)}$. $\mathrm{O}$ presente estudo confirma a acuidade visual preservada no Grupo SU II, com média de 20/50. Embora a média da acuidade visual do Grupo SU I tenha sido de 20/160, os testes estatísticos não paramétricos mostraram que não houve diferença estatística significante entre os dois grupos. Apenas um paciente com SU I obteve acuidade visual de movimentos de mão no olho esquerdo. A ocorrência de cegueira legal, como descrita anteriormente ${ }^{(12,18-19)}$ não foi encontrada nos grupos estudados.

Apesar do pequeno número de pacientes incluídos neste estudo e da diferença de participantes em cada grupo, a maioria dos casos teve diagnóstico prévio de síndrome de Usher tipo I (17/22 pacientes), contrariando alguns estudos que encontraram freqüência maior de síndrome de Usher tipo II $^{(13,20-21)}$.

O diagnóstico precoce da doença tem uma importância fundamental, principalmente, para a família dos pacientes, pois podem receber aconselhamento genético, evitando que outros membros da família nasçam com a síndrome, além de preparar os pacientes para uma vida social e econômica compatível com a sua capacidade visual.

\section{CONCLUSÃO}

Nos grupos estudados de síndrome de Usher tipo I e II, a acuidade visual mostrou-se relativamente preservada, sendo em média de 20/160 no grupo com SU I e de 20/50 no grupo com SU II. Os achados eletrorretinográficos mostraram-se severamente reduzidos em ambos os grupos, com a maioria dos pacientes apresentando respostas não detectáveis de bastonetes e cones. 


\section{ABSTRACT}

Purpose: Usher's syndrome (US) is a group of genetically distinct autossomal conditions, characterized by sensorineural hearing loss accompanied by a retinal dystrophy indistinguishable from retinitis pigmentosa (RP). The purpose of this study was to analyze full-field electroretinography (ERG) and visual acuity (VA) among patients with type I and II Usher's syndrome. Methods: Electroretinography responses and visual acuity were studied in 22 patients (mean age at test $=26.8 \pm 16.8$ years). Seventeen patients had SU type I and 5 patients were diagnosed as Usher's syndrome type II. Results: Mean visual acuity was $0.9 \log$ MAR $(20 / 160$, Snellen equivalent) for patients with Usher's syndrome type I and $0.4 \log$ MAR (20/50, Snellen equivalent) for patients with Usher's syndrome type II. Scotopic rod and maximal responses were non-detectable in both groups. Mean amplitude for oscillatory potentials was $14.5 \mu \mathrm{V}$ \pm 6.1 in Usher's syndrome type I and $12.6 \mu \mathrm{V} \pm 5$.2 in Usher's syndrome type II. Cone responses were non-detectable in $95 \%$ of the patients with Usher's syndrome I and in 100\% of patients with Usher's syndrome II. Mean amplitude for $30 \mathrm{~Hz}$ flicker photopic cone response was $3.1 \mu \mathrm{V} \pm 4.1$ for Usher's syndrome type I and $1.0 \mu \mathrm{V} \pm 0.6$ for type II with mean implicit time of $34.0 \mathrm{~ms} \pm 6.2$ (US I) and $35.8 \mathrm{~ms} \pm 3.1$ (type II). Conclusions: Visual acuity was relatively preserved in both groups, however Usher's syndrome II group showed better visual acuity results. Electroretinography findings were severely reduced in both groups, with most patients showing non-detectable rod and cone responses.

Keywords: Retinitis pigmentosa; Usher's syndrome; Electroretinography; Visual acuity; Electrophysiology; Retinal diseases; Visually impaired persons

\section{REFERÊNCIAS}

1. Abreu M, Chies MA, Abreu G. síndrome de Usher: novos conceitos. Arq Inst Penido Burnier. 1997;39(1):13-21.

2. Liarth JCS, Gonçalves EA, Gonçalves JO, Neiva DM, Leal FAM. síndrome de Usher: características clínicas. Arq Bras Oftalmol. 2002;65(4):457-61.
3. Traboulsi EI, Drack AV, Salama H. Pigmentary retinopathy and systemic disease. In: Traboulsi EI. Genetic diseases of the eye. New York: Oxford University Press; 1998. p.651-2.

4. Grupo Brasil de Apoio ao Surdocego e Múltiplo Deficiente Sensorial. Sem Luz e sem som: vencendo a barreira do isolamento. J AME. [periódico na Internet]. 2002;34 Jul/Ago. [citado em 2005 Jan 4]. Disponível em: http:// www.ame-sp.org.br/jor34.htm.

5. Young NM, Mets MB, Hain TC. Early diagnosis of Usher syndrome in infants and children. Am J Otol. 1996;17(1):30-4.

6. Abraham FA, Cohen D, Sohmer H. Usher's syndrome: electrophysiological tests of the visual and auditory systems. Doc Ophthalmol, 1977;44(2):435-44.

7. Birch DG. Clinical electroretinography. In: Fuller DG, Birch DG, eds. Assessment of visual function for the clinician. Ophthalmol Clin North Am. 1989; 2:469-98.

8. Scholl HP, Zrenner E. Electrophysiology in the investigation of acquired retinal disorders. Surv Ophthalmol. 2000;45(1):29-47.

9. Niemeyer G. From the symptom to electroretinography diagnosis. Klin Monatsbl Augenheilkd. 1999;214(5):328-31.

10. Edwards A, Fishman GA, Anderson RJ, Grover S, Derlacki DJ. Visual acuity and visual field impairment in Usher syndrome. Arch Ophthalmol. 1998; 116(2):165-8.

11. Szlyk JP, Fishman GA, Master SP, Alexander KR. Peripheral vision screening for driving in retinitis pigmentosa patients. Ophthalmology. 1991;98(5): 612-8.

12. Grover S, Fishman GA, Alexander KR, Anderson RJ, Derlacki DJ. Visual acuity impairment in patients with retinitis pigmentosa. Ophthalmology. 1996;103(10):1593-600.

13. Tsilou ET, Rubin BI, Caruso RC, Reed GF, Pikus A, Hejtmancik JF, et al. Usher syndrome clinical types I and II: could ocular symptoms and signs differentiate between the two types? Acta Ophthalmol Scand. 2002;80(2):196-201.

14. Pereira JM, Mendieta L, Sacai PY, Salomão SR, Berezovsky A. Estudo normativo do eletrorretinograma de campo total em adultos jovens. Arq Bras Oftalmol. 2003;66(2):137-44.

15. Mets MB, Young NM, Pass A, Lasky JB. Early diagnosis of Usher syndrome in children. Trans Am Ophthalmol Soc. 2000;98:237-42.

16. Edwards A, Fishman GA, Anderson RJ, Grover S, Derlacki DJ. Visual acuity and visual field impairment in Usher Syndrome. Arch Ophthalmol. 1998;116 (2): $165-8$

17. Fishman GA. Usher's Syndrome: visual loss and variations in clinical expressivity. Perspect Ophthalmol. 1979;3:97-103.

18. Seeliger M, Pfister M, Gendo K, Paasch S, Apfelstedt-Stylla E, Plinkert P, et al. Comparative study of visual, auditory and olfactory function in Usher Syndrome. Graefer Arch Clin Exp Ophthalmol, 1999;237(4):301-7.

19. Grover S, Fishman GA, Anderson RJ, Tozatti MS, Heckenlively JR, Weleber RG, et al. Visual acuity impairment in patients with retinitis pigmentosa at age 45 years or older. Ophthalmology. 1999;106(9):1780-5.

20. Otterstedde CR, Spandau U, Blankenagel A, Kimberling WJ, Reisser C. A new clinical classification for Usher's Syndrome based on a new subtype of Usher's Syndrome type I. Laryngoscope. 2001;111(1):84-7.

21. Bereketoglu M, Avsar EU, Turan O, Afrashi F, Apaydin F. The incidence of Usher's Syndrome and its clinical types. Kulak Burun Bogaz Ihtis Derg. 2002;9(1):15-20.

\section{Ao enviar um artigo para publicação, leia ATENTAMENTE as instruções para autores, constante no final de cada fascículo.}

